\title{
Editorial: Behavioral Medicine in the Asia Pacific
}

\author{
Akihito Shimazu $^{1} \cdot$ Akizumi Tsutsumi $^{2} \cdot$ Kazuhiro Yoshiuchi $^{1}$
}

Published online: 25 May 2016

(C) International Society of Behavioral Medicine 2016

The Asia Pacific is the world's most populous region, which is diverse culturally, economically, and socially. Although the dominant theories and techniques in behavioral medicine have been developed and refined in western cultures, little attention has been given to research and practice of behavioral medicine in the region and, hence, more research is needed to examine whether they are applicable to the Asia Pacific. This special issue is intended to improve the communication and collaboration between Asia Pacific researchers and practitioners in the field of behavioral medicine and colleagues elsewhere in the world.

In the response to the call for contributions to a special issue of the International Journal of Behavioral Medicine in September 2014, 36 manuscripts were submitted. We are pleased to present the special issue on Behavioral Medicine in the Asia Pacific, consisting of 15 interesting papers: five papers on public health from Taiwan and Japan; six papers on clinical and psychosomatic medicine from China, Japan, and the UK; and four papers on psychological and behavioral perspectives from Japan, Singapore, Taiwan, and the USA.

In the first part of the special issue, five papers on public health were contributed from Taiwan and Japan.

Using large national datasets, Liao, Tsai et al. showed that daily traveling by private motorized vehicle was associated with poor performance in abdominal muscular strength and endurance in 52,114 Taiwanese adults. The findings provide the insight that daily private motorized-vehicle travelers are

Akihito Shimazu

ashimazu@m.u-tokyo.ac.jp

1 The University of Tokyo, Bunkyo-ku, Tokyo, Japan

2 Kitasato University, Sagamihara, Kanagawa, Japan considered as an important target population to improve nation-wide physical fitness.

In another contribution by Liao, Shibata et al., sufficient physical activity ( $\geq 150 \mathrm{~min} /$ week) and some combinations of physical activity and sedentary behaviors were shown to be associated with lower risk of depressive symptoms. Although the data were collected by an internet survey, the findings suggest meeting physical activity recommendation would be beneficial for mental health and encourages further investigation on the issue.

In the third contribution from Japan, upstream factors that could determine physical activity level were also examined. A path analysis was conducted to elucidate the association among work environment, employees' attitude for the work and exercise habits, and coronary risk factors (Watanabe et al.) The findings suggest that job resources lead to favorable lipid profiles through workers' vigor and exercise habits.

In Japan, suicide prevention is one of the important issues in public health. A multilevel analysis of Japanese large data of 42,499 workers revealed that the risk of suicidal ideation among Japanese workers was associated not only with job demands and job resources at the employee-level but also coworker support in the organization level (Otsuka et al.). The findings provide important implications for interventional targets to prevent suicide in Japan, where over 6000 workers commit suicide every year.

Another contribution from Japan is related to innovation of psychosocial measurement. Based on item response theory, Iwata et al. developed a computerized-adaptive testing (CAT) technique to measure mental health status of workers (Iwata et al.). The merit of using the technique - shortening the measurement and evaluation time, avoiding the disadvantage due to remembering the items, etc.-would make the CAT a useful measurement tool for stress check at workplace. 
In the second part of the special issue, six papers on clinical and psychosomatic medicine were contributed from China, Japan, and the UK.

Chan et al. investigated the association between affect, affective variability, and self-reported health status in a large representative sample of adults in China. The results showed that affective variability was negatively related to subjective health conditions and positively related to diagnosed illness status, after controlling for individuals' mean levels of affect and demographic variables.

Ochi et al. investigated the association between parental social interaction (both received and provided social support) and offspring behavior problems. They found that higher maternal social interaction is significantly associated with lower odds of both difficult and prosocial behavior problems, while the same associations were not found for paternal social interaction. Further, provided maternal social support showed an independent negative association with prosocial behavior problems in offspring, even when adjusted for received maternal social support and paternal social interaction.

$\mathrm{Xu}$ et al. investigated associations between styles of parental bonding and detailed aspects of primary dysmenorrhea among Chinese university-student women. They found that inappropriate parental bonding or chronic traumatic attachment styles have relationships respectively with functional and emotional disturbances due to primary dysmenorrhea.

Hirokawa et al. investigated whether changes in job demands could modify associations between changes in testosterone levels and andropause symptoms in male Japanese workers, in a longitudinal study. The study showed that andropause symptoms might be affected by changes in testosterone levels and job demands. Change in job demands may modify associations between changes in testosterone levels and andropause symptoms.

Yang et al. reported a systematic review of psychological treatments for chronic pain in adult populations of East Asia and Southeast Asia. They found that a majority of the studies were rated as weak in design quality because the current available literature on psychological treatments for chronic pain in East and Southeast Asia is generally small in scale, mostly preliminary.
Suzuki et al. investigated the acceptability of a touch screen tablet survey among cancer patients in Japan; touch screen computer surveys are already an acceptable mode of collecting information about cancer patients' wellbeing in Western clinical settings. The conclusion was that selfadministered touch screen tablet surveys may not be entirely appropriate for older cancer patients, or possibly for patients with lower educational attainment, although patient selfreported acceptability of the touch screen survey was high.

In the third part, psychological and behavioral perspectives were addressed by four papers from Japan, Singapore, Taiwan, and the USA.

Watanabe and Yamauchi examined the association between psychosocial factors of overtime work and worknonwork balance among Japanese nurses. Using a multilevel structural equation modeling (SEM) technique, they demonstrated that involuntary overtime work has a negative effect on work-nonwork balance, and voluntary overtime work had a positive direct effect but a negative indirect effect.

Chen et al. examined the effects of an 8-week aquatic exercise program on objectively measured sleep parameters among older adults with mild sleep impairment. They revealed that the program had significant benefits on some sleep parameters, including shorter sleep onset latency and better sleep efficiency.

Lim et al. explored the course and determinants of quality of life (QOL) over 12 months in peritoneal dialysis (PD) patients. Although PD offered the convenience of home-based care, it was associated with persisting QOL impairments and diminishing QOL over time, especially in domains related to quality of care and support.

Finally, Roberson et al. described the prevalence of depression, anxiety, pharmaceutical treatment, and help-seeking behaviors among a multiethnic population of women with recent live births in Hawaii. They covered multiple indicators related to depression and anxiety beginning in the year prior to pregnancy and extending into the postpartum period, and illustrate the complexity of the mental health issues surrounding pregnancy in Hawaii.

We thank all our authors for their contributions. We hope this special issue has been of value to and interesting for both researchers and practitioners in behavioral medicine, both in the Asia Pacific region and elsewhere. 